Revista de Psicología de la PUCP. Vol. XVII, 1, 1999.

\title{
EL DESARROLLO HUMANO COMO PROCESO PROBABILISTICO: LECCIONES DE TREINTA AÑOS DE ESTUDIOS SOBRE EL DESARROLLO INFANTIL EN EL TERCER MUNDO ${ }^{1}$
}

\author{
Ernesto Pollitt ${ }^{2}$ \\ Universidad de California, Davis
}

La tesis de este trabajo es que las predicciones sobre la dirección del desarrollo humano basándose en un solo evento ocurrido durante los primeros años de la vida generalmente tienen una débil validez interna. Las limitaciones de dichas predicciones no se deben a las limitaciones inherentes a los estudios que han puesto a prueba la validez de la predicción sino a una conceptualización errada sobre la naturaleza misma del desarrollo. Este no esta determinado por efectos principales sino por complejas relaciones recíprocas entre los diferentes componentes del organismo y entre este y el ambiente físico y social. Se propone que hay que modelar el desarrollo como un proceso probabilistico cuya trayectoria se va forjando gradualmente.

Palabras claves: desarrollo humano, proceso probalístico, desarrollo del niño, Tercer Mundo.

Human development as a probabilistic process: lessons from thirty years working on child development in the Third World

The thesis of this paper is that the predictions of later human development based on a single event during early life generally have a weak internal validity. These limitations are not due to problems of study design but to un erroneous conceptualization of the very nature of development. This is not determined by main effects but by the complex relationships among domains within the organism and between the organism and the physical and the social environment. The propasal is to model human development as a probabilistic process that gradually shapes its developmental trajectory. Key words: human development, probabilistic, process, child development, Third World.

1. Trabajo presentado en la reunión convocada por la Pontificia Universidad Católica del Perú con motivo del nombramiento del autor como Profesor Honorario del Departamento de Humanidades. Lima, 17 de noviembre de 1998.

2. Doctor en Psicología. Profesor del Departamento de Pediatría de la Escuela de Medicina de la Universidad de California, Davis. En 1999 recibió el premio por excelencia en Investigación en Nutrición Internacional de la Sociedad Americana de Nutrición. Ha publicado varios libros y artículos científicos. Dirección: TB 139, Department of Pedriatrics, University of California, Davis. CA.95616. CE:epollit@ucdavis.edu. 
El propósito de mi trabajo es ilustrar cómo la investigación de los efectos funcionales de la desnutrición ha contribuido al conocimiento científico de los procesos y mecanismos que forjan la ontogénesis humana. De manera específica, voy a discutir la idea de las determinantes principales en la ontogenia conductual, que implica la continuidad en el desarrollo y la posibilidad de predecir el futuro con información presente. Voy a cuestionar esta idea para concluir afirmando que el desarrollo no es continuo, sino que es un proceso probabilístico (Gottlieb, 1992; Gottlieb, Whalsten y Lickliter, 1998; Horowitz, 1994). La charla está basada en un artículo que publiqué en 1988 y en los resultados de estudios longitudinales en los que he participado desde entonces (Pollitt, 1988; Pollitt, Gorman, Engle, Martorell y Rivera, 1993; Pollitt, Watkins y Husaini, 1997). Creo, además, que dichos resultados han aumentado la validez de lo propuesto.

Con fines didácticos comenzaré compartiendo algunas definiciones elementales sobre la desnutrición. Su causalidad inmediata es una dieta cualitativa y cuantitativamente deficiente, acompañada por enfermedades infecciosas frecuentes que disminuyen el apetito. A otro nivel de la cadena causal, la desnutrición es el resultado de recursos económicos familiares que no satisfacen las necesidades básicas, así como de situaciones sociopolíticas conflictivas. Sus efectos son moeltiples: retarda el crecimiento físico y el desarrollo de órganos vitales, rezaga el desarrollo motor, debilita la resistencia a las infecciones, disminuye la actividad física, interfiere con la regulación emocional, limita las interacciones con el ambiente y atrasa el funcionamiento cognitivo. Estos efectos no son independientes losunos-de-los-otros sino interactivos; y los efectos de las interacciones son muchas veces superiores a los efectos particulares. Además, la desnutrición infantil en la generalidad de los casos no es un proceso agudo (v.g. deshidratación) sino crónico. Con frecuencia comienza durante la gesta- 
ción o brevemente después del nacimiento y se mantiene con distinta intensidad hasta el segundo o tercer año de vida. Esto no niega que la desnutrición también afecte a otras edades durante periodos fisiológicos particulares (v.g. gestación y lactancia).

La desnutrición no es una entidad clínica con un conjunto de síntomas y un perfil bioquímico que la caractericen. Esto se ve con claridad en los casos leves o moderados, que representan el porcentaje más alto de casos de desnutrición en el mundo. Además, la deficiencia proteico-energética óla más frecuenteó no existe aisladamente sino que por lo general está asociada a deficiencias de otros nutrientes (v.g. hierro y zinc). Este síndrome pluricarencial tiene una incidencia tan alta en algunos países del Africa (sobre todo al sur del Sahara), en la India, Pakistán, Bagladesh, en el sudeste asiático, en Centro y Sudamérica, que el desarrollo del niño desnutrido es normativo en ese contexto ${ }^{3}$.

\section{Determinantes principales y explicaciones lineales}

La mayor parte de los estudios sobre efectos de la desnutrición antes y después del nacimiento en el desarrollo psicobiológico del niño se guió por la idea de determinantes principales que en biología relaciona un factor causal con un efecto específico en una relación de uno-a-uno. En el contexto del crecimiento y el desarrollo humano, esta idea se asocia a la teoría de los gérmenes en medicina y salud pœblica (Susser y Susser, 1996. Originalmente, tal teoría proponía que la causa exclusiva de una enfermedad infecciosa es un virus o una bacteria que contamina al organismo. $\mathrm{Si}$ bien la teoría ha cambiado a través del tiempo y ha considerado la inclusión de factores moderadores de dicha relación, hasta hoy se mide, por ejemplo, la intensidad de una infección parasítica intestinal por el nœmero de huevos del parásito dentro de un gramo de material fecal. Con el predominio de la epidemiología de las enfermedades crónicas y la consideración de los llamados factores de riesgo, la teoría del germen dio paso a modelos

3. Definiendo como 'normativoí la tendencia central de una población. 
El desarrollo humano como proceso probabilístico: lecciones de treinta años de...

multicausales. Sin embargo, la emergencia de nuevas enfermedades infecciosas, en particular la del síndrome de inmunodeficiencia adquirida (SIDA), ha hecho resaltar nuevamente la conceptualización de la teoría.

Pasemos ahora al caso de la desnutrición, teniendo presente que, como dije antes, no es una entidad clínica ni está caracterizada por un conjunto de síntomas específicos. En el contexto de los estudios sobre la desnutrición y el desarrollo psicobiológico, la idea de determinantes principales se puede representar con la siguiente ecuación de dos variables:

\section{Desviación de un criterio de referencia = $f$ (Riesgo nutricional)}

Si por riesgo nutricional entendemos la naturaleza, intensidad, duración y temporalidad de la desnutrición, esta ecuación se puede operacionalizar y ejemplificar de la siguiente manera:

$\%$ Disminución del $C I=f$ (desnutrición proteico-energética moderada, durante los primeros 24 meses después del nacimiento)

Figura 1. Desviación de un criterio de referencia.

Este tipo de planteamiento se puede trasladar fácilmente a la econometría y es de particular interés para organismos como el Banco Mundial. Si tal formulación es correcta, entonces se podría calcular el producto de un préstamo de $\mathrm{X}$ cantidad de dólares para subir el coeficiente intelectual en $\mathrm{X}$ cantidad de puntos mediante el aumento de $\mathrm{X}$ kilocalorías en el consumo energético de niños de los 6 a los 24 meses.

Un correlato muy cercano a esta idea de determinantes del desarrollo es la noción de periodos críticos. Por ello entiendo de manera selectiva los periodos o ventanas del desarrollo dentro de los cuales el estar expuesto a un factor de riesgo biológico ${ }^{4}$ produce cambios cerebrales que, a su vez,

4. Defino aquí como 'riesgo biológicol las probabilidades de un individuo de tener una enfermedad o un cambio en su salud durante un periodo de tiempo específico. Los factores de riesgo biológico 
determinan una anomalía funcional permanente. Este concepto de periodos críticos ha sido validado definitivamente en embriología. Sabemos, por ejemplo, que la varicela en una mujer que está en el primer trimestre de gestación determina malformaciones congénitas irreversibles. En el contexto presente, sin embargo, no estamos hablando sobre principios moleculares sino sobre la aplicabilidad del concepto de periodos criticos en el desarrollo cognitivo.

En el contexto específico del estudio de los efectos de la desnutrición temprana, la idea de periodos críticos y la evidencia de laboratorio llevó a la hipótesis de que la desnutrición de energía y proteína durante los periodos de mayor aceleración en el crecimiento cerebral (primeros 24 meses de vida después del nacimiento) tenía un efecto irreversible (Dobbing y Sands, 1979; Winick y Noble, 1966; Winick y Rosso, 1969). Esta hipótesis llevaba implícita la idea de que la desnutrición era un factor suficiente para producir el retardo mental. Una extensión natural de esta idea fue que la desnutrición que se presenta después del periodo crítico no deja secuela.

Varios estudios experimentales e investigaciones patológicas básicas reforzaron las ideas de determinantes principales y periodos críticos. Estudios con ratas mostraron, por ejemplo, que la desnutrición experimentalmente inducida durante el periodo prenatal y durante el periodo de lactancia (hasta los 21 días) retardaba el proceso de proliferación de todas las células cerebrales y demoraba la migración de células y el proceso de mielinización (Dobbing, 1990; Levitsky y Strupp, 1995). Estos estudios en animales encontraron su corroboración en el desarrollo humano a través de investigaciones cerebrales en niños que habían muerto por una desnutrición severa y mostraban reducciones dramáticas en el contenido de ARN y ADN en todo el cerebro (Winick y Rosso, 1969).

son las circunstancias o eventos que ocurren durante el embarazo $(\nu . g$. crecimiento intrauterino retardado), el periodo perinatal ( $\nu . g$. hipoxia) o la infancia ( $\nu . g$. intoxicación con plomo), que incrementan las probabilidades de desviar la trayectoria del desarrollo de un niño fuera del camino a seguir cuando las necesidades fisiológicas y emocionales básicas son satisfechas. 
El desarrollo humano como proceso probabilístico: lecciones de treinta años de...

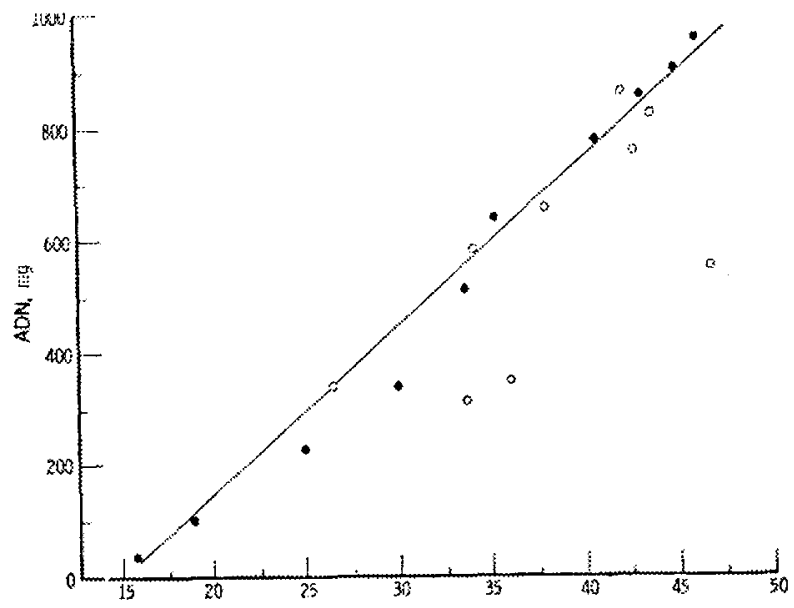

Perímetro Cefálico, cm.

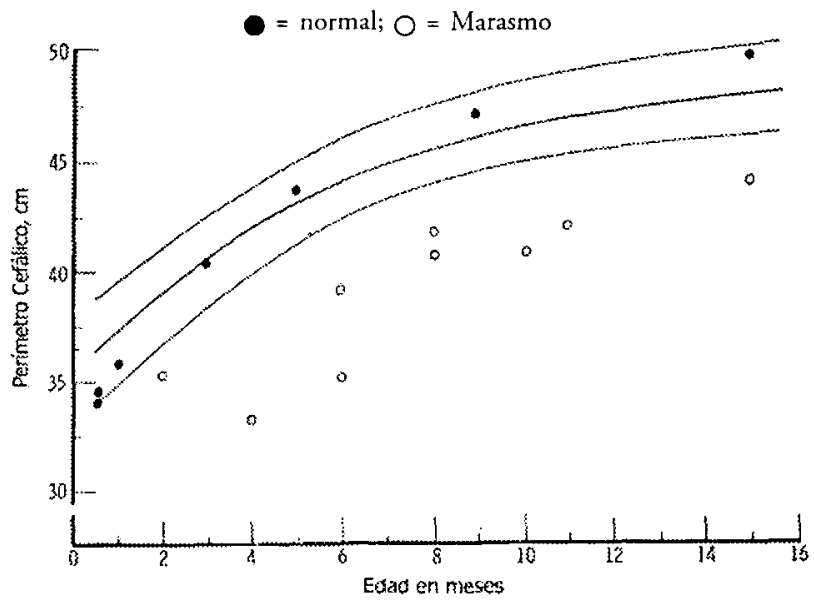

= normal; $O=$ Marasmo, las líneas indican valores normales de los Estados Unidos.

Figura 2. ADN cerebral vs perímetro cefálico y perímetro cefálico vs. edad.

Fuente: Winick M. y Rosso P. (1969) "Head circunference and cellular growth of the brain in normal and marasnic children". The Journal of Pediatrics, 74, 5, 774-778. 
La idea de determinantes principales y la conceptualización de periodos críticos, así como los datos experimentales con modelos animales, llevaban a la conclusión de que en los niños desnutridos la relación entre la deficiencia de macronutrimentos y las consecuencias funcionales era mediada por cambios en la arquitectura cerebral.

\section{Imprecisión en la idea de determinantes principales}

Como tanto se ha dicho, el tiempo lleva al cambio de paradigmas en la ciencia (Kuhn, 1962). Gradualmente, varios investigadores comprendieron que la conceptualización de un efecto lineal no conducía a una comprensión cabal de los efectos de la desnutrición entre niños que viven en condiciones de pobreza extrema (Brown y Pollitt, 1997; Gorman, 1995; Grantham McGregor, 1995; Wachs, 1995). Se hizo evidente que la desnutrición tiene un origen multicausal y se reconoció que el problema era demasiado complejo para reducirlo a la medición de un efecto principal. Paso a discutir tres argumentos en particular.

1) Efectos de la desnutrición primaria y secundaria.

2) Efectos del ambiente y de las características y experiencias individuales.

3) Efectos de las intervenciones uni- y multifocales.

\section{Argumentos}

\section{Efectos de la desnutrición primaria y secundaria}

Los resultados de los estudios sobre el desarrollo de niños con una historia de desnutrición que viven en condiciones de pobreza extrema contradicen los resultados de los estudios de niños con una historia de desnutrición producida por un problema médico como, por ejemplo, la estenosis pilórica ${ }^{5}$ o los síndromes de malabsorción. En el caso de la

5. Estenosis pilóricaí es una reducción del calibre del píloro. 
El desarrollo humano como proceso probabilístico: lecciones de treinta años de...

desnutrición primaria, el funcionamiento del niño está disminuido. Opuestamente, en los casos de desnutrición secundaria a un desorden fisiológico, hay concordancia entre el nivel de desarrollo y el potencial del niño (Berglund y Rao, 1973; Klein, Forbes y Nader, 1975). Es decir, la desnutrición moderada fuera del contexto de la pobreza por lo general no deja secuela en el desarrollo intelectual.

Esta contradicción puede tener, entre otras, dos explicaciones. La primera es que la desnutrición no afecta el desarrollo psicobiológico y que la disminución intelectual observada en los niños desnutridos en condiciones de pobreza se debe a la pobreza y no a las deficiencias de nutrientes. Es decir, los factores sociales de la pobreza confunden la relación entre desnutrición y rendimiento cognitivo. La explicación alternativa es que las condiciones ambientales en niños con una historia de desnutrición con etiología médica protegen al niño, previniendo que la desnutrición deje un daño permanente. Hoy sabemos que la segunda de estas dos explicaciones es válida.

\section{Efectos del ambiente $y$ de la experiencia individual}

Durante los oltimos veinte años aproximadamente, varios estudios han mostrado que, con excepciones clínicas, las probabilidades de una secuela funcional ( $v . g$. problemas de aprendizaje) entre niños que sufrieron el mismo trauma biológico ( $v \cdot g$. retardo en el crecimiento intrauterino) es una función negativa de las características sociales y económicas de la familia. Las probabilidades de que se encuentren problemas de aprendizaje escolar entre niños que nacieron con un peso $<2,500$ gramos, por ejemplo, varían en función directa de su nivel económico: esto es, las probabilidades aumentan conforme baja el nivel económico.

Muchos estudios sobre desnutrición y funcionamiento cognitivo concuerdan con las observaciones del desarrollo psicobiológico de niños expuestos a otros factores de riesgo biológico (v.g. bajo peso al nacer). Se ha demostrado que, aun en comunidades en condiciones de pobreza absoluta, los factores socioeconómicos predicen la variabilidad del desa- 
rrollo mental de niños con una historia de malnutrición temprana. Los factores socioeconómicos, sin embargo, no son los œnicos que actœan como moderadores.

En un estudio en cuatro aldeas rurales en El Oriente (Guatemala) se demostró que los beneficios de un suplemento alimentario rico en energía y proteínas para el desarrollo intelectual de niños en riesgo nutricional, variaban en función de las características sociales y económicas de esos niños. Los participantes fueron todos los niños de 7 años o menos que vivían en las aldeas en el momento del inicio del estudio, en 1969; todos los niños que se mudaron a las aldeas durante el proyecto; y todos los niños nacidos en las aldeas entre el 1 de enero de 1969 y el 28 de febrero de 1977. Originalmente, más de dos mil niños y sus madres, provenientes de cuatro aldeas, participaron en la intervención nutritiva. El estudio incluyó dos tipos de alimento suplementario: el atole, rico en energía y proteínas, y el «fresco», con un modesto valor energético y ningún valor proteico.

En 1988/89 tuve la oportunidad de dirigir el estudio (Pollitt, Gorman, Engle, Martorell y Rivera, 1993) de seguimiento psicoeducacional de estos sujetos, cuando sus edades variaban entre los 11 y los 26 años de edad. En esta nueva fase de la investigación hubo una cobertura del $80 \%$ de los sujetos originales. En efecto, se administró una batería de pruebas psicológicas y educacionales a 1,411 sujetos. La batería incluía pruebas de alfabetismo, habilidad numérica, lectura y conocimientos generales, así como pruebas estandarizadas de logros en lectura y vocabulario, y las matrices progresivas de Raven. 


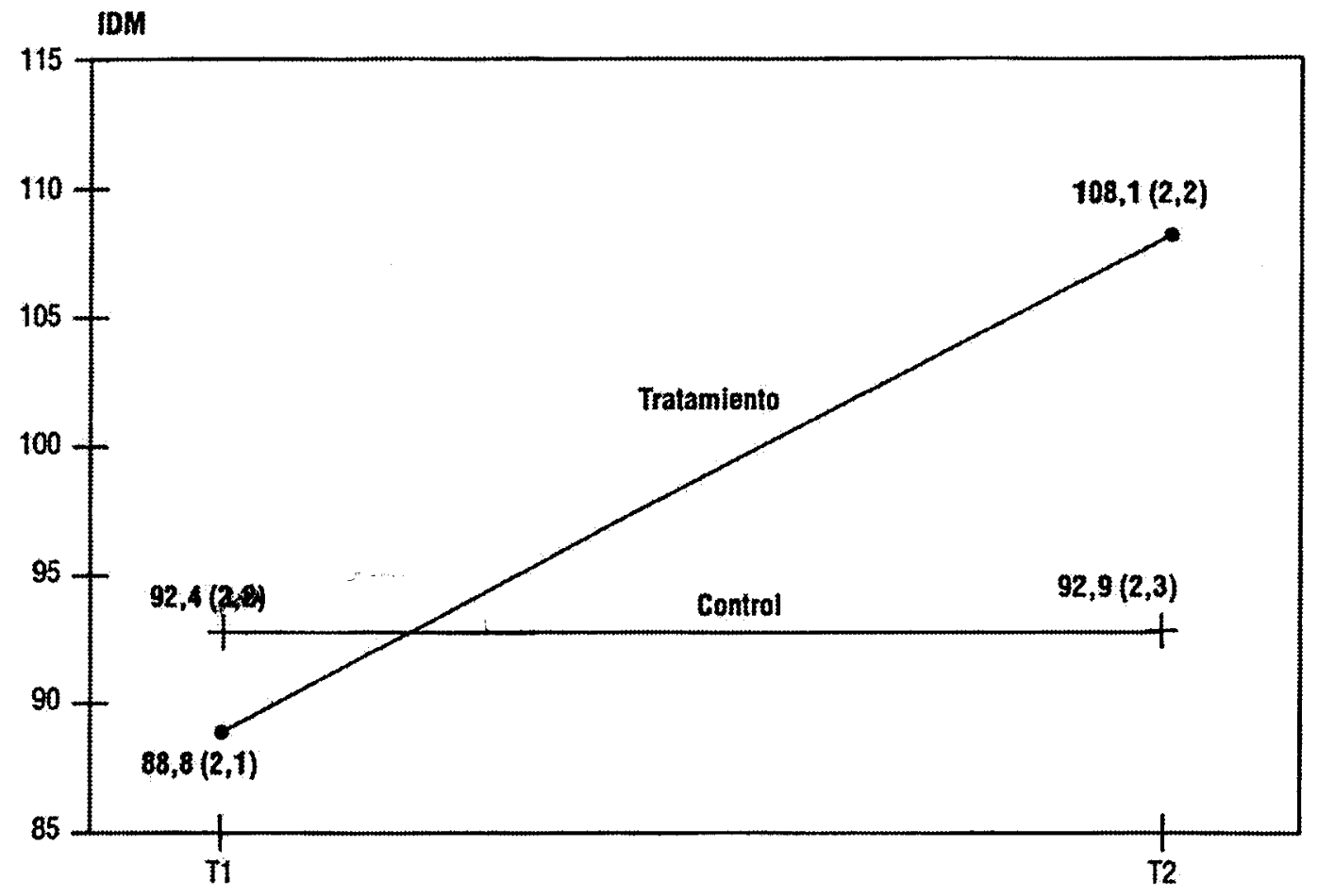

Nota. Puntaje de Desarrollo Mental (IDM): Media antes del tratamiento (T1) y después del tratamiento (T2).

Figura 3. Interacción del alimento adicional y el nivel socioeconómico en el estudio de El Oriente en

Guatemala. Fuente: Pollit R. y Gorman K. (1997) Carencias nutricionales como factores de riesgo en el desarrollo infantil. En: Nutrición $\vec{\omega}$ y alimentación en los primeros años de vida. PALTEX, pp. 309-334. 
Los resultados de los análisis fueron considerablemente consistentes. La Figura 3 es ilustrativa. Como se puede ver, en el grupo que recibió alimentación adicional rica en proteína y energía no hay diferencia en el desarrollo del lenguaje de los niños, en función del nivel de pobreza. En el grupo que recibió el fresco, el nivel de pobreza sí afectó dicho desarrollo: resultó mucho menor entre los niños que vivían en condiciones de pobreza extrema, en comparación con los que vivían en condiciones de pobreza leve.

La conclusión es que incluso cuando la desnutrición ocurra tempranamente, ella no es una condición suficiente para fijar la trayectoria del desarrollo. La corrección de una desviación del desarrollo está determinada en parte por las circunstancias ambientales y por las experiencias individuales. Bajo las condiciones a las cuales me estoy refiriendo, el organismo tiene la capacidad de modificar la dirección de un desarrollo desviado.

Por otro lado, las probabilidades de seguir en una trayectoria desviada aumenta conforme el niño crece y continœea expuesto a condiciones ambientales que no pueden satisfacer las necesidades fisiológicas, emocionales y educacionales básicas. La evidencia que proviene de distintas fuentes confirma la llamada hipótesis del efecto acumulativo. Carmen Saco y yo comparamos, por ejemplo, la relación entre el nivel de aprendizaje y la edad de niños de dos comunidades (La Huaca y Los Llanitos) rurales de extrema pobreza localizadas entre el pueblo de Escuintla y el Puerto de San Jose en Guatemala y niños de Cambridge, Massachusetts (Saco-Pollitt, Pollitt y Greenfield, 1985). En Guatemala, los niños mayores rendían al mismo nivel que los niños menores. Es decir, la edad no moderaba la relación entre las dos variables. En Cambridge, los resultados fueron totalmente opuestos: allí la edad era un componente fundamental en dicha relación; el aprendizaje variaba en función directa de la edad de los niños.

Lamentablemente, las condiciones ambientales por lo general no cambian tan rápido como es necesario para que el desarrollo del niño esté expuesto a circunstancias que puedan corregir el desvío en su desarrollo proveniente de la exposición a la desnutrición y a otros factores de pobreza. 
El desarrollo humano como proceso probabilístico: lecciones de treinta años de...

\section{Resultados de intervenciones unifocales y multifocales}

La tercera razón que nos lleva a la crítica del modelo de dos variables es la diferencia entre los resultados de estudios de alimentación suplementaria con y sin componentes de salud y educación.

La alimentación suplementaria de la madre gestante y lactante y de los niños durante los dos primeros años de vida ha producido efectos estadísticamente significativos pero relativamente modestos. Por el contrario, intervenciones multifocales que además de tener un programa de alimentación suplementaria tenían también los componentes de salud y educación, han dado resultados con mayor rédito. Esto se puede observar al hacerse las comparaciones pertinentes con estudios llevados a cabo en Bogotá, Colombia; Guatemala; Harlem, Nueva York; y Sui Lin, Taiwán (Pollitt y Oh, 1994). La consistencia de estos estudios es saltante si consideramos que la selección de las muestras, el tipo de suplemento alimentario, las escalas de medición psicológica y la edad en que fueron evaluados los niños variaba entre los estudios. Estas diferencias entre los efectos de las intervenciones uni- $y$ multifocales nos llevan a concluir que la interacción entre factores que afectan el desarrollo del niño tiene un efecto sinérgico.

\section{Conclusiones}

La comparación de los resultados provenientes de niños desnutridos dentro y fuera del contexto de pobreza; los análisis sobre los efectos moderadores del nivel socioeconómico en el contexto de poblaciones que viven en la pobreza; y la comparación de los efectos de las intervención uni- y multifocales, nos llevan a concluir que la idea de determinantes principales da una visión parcial o equívoca de lo que sucede.

Las limitaciones en la predicción del futuro basándose en información presente no es un problema de diseño ${ }^{6}$. La información sobre la desnutrición

6. El modelo -especificamente en el contexto de las investigaciones sobre el desarrollo humanono considera dos características fundamentales del organismo que contribuyen a determinar el curso duradero del desarrollo. Me refiero específicamente a la plasticidad y la canalización*. 


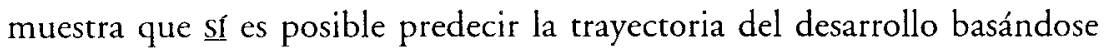
en el conocimiento de un solo evento biológico o psicológico presente durante la infancia; pero las probabilidades de una predicción internamente válida son muy limitadas (Pollitt, Watkins y Husaini, 1997). Dicho de otra forma, el margen del error es muy alto. Lo que hemos encontrado es que el aumento del poder de predicción requiere de conocimientos sobre lo ocurrido en el desarrollo anterior y posterior al evento que se estudia. Ello nos obliga a plantear afirmaciones condicionales como la siguiente:

$$
\begin{gathered}
Y=f(x)-\text { si es que }-A \text { está presente y } B \text { está ausente } \\
\mathrm{A}=\text { variable que promueve el desarrollo } \\
\mathrm{B}=\text { variable que limita el desarrollo }
\end{gathered}
$$

Figura 4

El desarrollo se modela, entonces, como un proceso probabilístico dentro del cual se incorporan factores internos y externos al organismo, continuamente activos en la moderación de la trayectoria a seguir (SacoPollitt et al. 1985; Horowitz, 1994; Sameroff, 1994). Estos factores pueden mantener el efecto de un trauma ocurrido tempranamente en la infancia; pero también pueden inducir o fortalecer cambios en la dirección de dicha trayectoria, aumentando o disminuyendo la distancia en relación con una población referente. Lo que quiero enfatizar es que la influencia del contexto ambiental en el desarrollo cognitivo del niño hace que las probabilidades del curso del desarrollo varíen en función de la naturaleza de lo que ocurre en los distintos estadios de desarrollo. La conclusión a la que he llegado a través de los resultados de varios estudios sobre el desarrollo de niños desnutridos encaja con formulaciones contemporáneas sobre el desarrollo ontogenético del ser humano.

Plasticidad es la noción de que, dentro de los límites de las diferencias y características estructurales interindividuales, el organismo tiene la maleabilidad o flexibilidad necesaria para adaptarse a circunstancias o eventos nuevos a los cuales está expuesto. Por canalización se entienden las características del organismo para sortear o lidiar con estrés ambiental antes de desviarse de la trayectoria trazada por la especie. (* MC CALL 1981.) 
El desarrollo humano como proceso probabilístico: lecciones de treinta años de...

\section{Referencias}

Berglund, G. y Rao, E. (1973). A long-term follow-up investigation of patients with hypertrophic pyloric stenosis, with special reference to the physical and mental development. Acta Paediatrica Scandinavica, $62,125$.

Brown, L. y Pollitt, E. (1997). Malnutrition, Poverty and Intellectual Development. Scientific American, 274(2), 26-31.

Dobbing, J. (1990) Vulnerable periods in developing brain En J. Dobbing (Ed.), Brain, behaviour and iron in the infant diet (pp. 1-26). Nueva York: Springer Verlag.

Dobbing, J. y Sands, J. (1979). Comparative aspects of the brain growth spurt. Early Human Development, 3, 79-83.

Gorman, K. (1995). Malnutrition and cognitive development: Evidence from experimen-tal/quasi-experimental studies among the mild-tomoderate malnourished. Journal of Nutrition, 125, 2239A-2244S.

Gottlieb, G. (1992). Individual development and evolution: the Genesis of Novel Behavior. Nueva York: Oxford University Press.

Gottlieb, G., Wahlsten, D. y Lickliter, R. (1998). The significance of biology for human development: A developmental psychobiological systems view. En W. Damon y R.M. Lerner (Eds.), Handbook of child psychology. Theoretical models of human development (pp. 233-273). Nueva York: Wiley.

Grantham-McGregor, S. (1995). A review of studies of the effects of severe malnutrition on mental development. Journal of Nutrition, 125, 2233S-2238S.

Horowitz, F. D. (1994). Developmental theory, prediction and the developmental equation in folow-up reseafch. En S.L. Friedman y H.C. Haywood (Eds.), Developmental follow-up. Concepts, domains, and methods (pp. 27-44). Nueva York: Academic Press.

Klein, P. S. Forbes, G. B. y Nader, P. R. (1975) Effects of starvation in infancy (pyloric stenosis) on subsequent learning abilities. Jounal of Pediatrics, 87, 8. 
Kuhn, T. S. (1962). The structure of scientific revolutions. Chicago: University of Chicago Press.

Levitsky, D. A. y Strupp, B. J. (1995). Malnutrition and the brain: Changing concepts, changing concerns. Journal of Nutrition (Suplemento). 125, 2212S-2221S.

Mc Call, R. B. (1981). Nature-nurture and the two realms of develpment: a proporsed integration with respect to mental development. Child Development, 52, 1-12.

Pollitt, E. (1988). A critical view of three decades of research on the effects of chronic energy malnutrition on behavioral development. En Schurch y Scrimshaw (Eds.), Chronic energy deficiency: Consequences and related issues (pp. 77-94). Lausana, Suiza: Nestle Foundation.

Pollitt, E. y Oh, S. (1994). Early suplementary feeding, child development and health policy. Food and Nutrition Bulletin, 15, 208-214.

Pollitt, E., Gorman, K, Engle, P, Martorell, R. y Rivera, J. (1993). Early supplemen-tary feeding and cognition. Effects over two decades. Monographs of the Society for Research in Child Development, 58 (7).

Pollitt, E., Watkins, W. y Husaini, M. (1997). Three-month nutritional supplementation in Indonesian infants and toddlers benefits memory function 8 and later. American Journal of Clinical Nutrition, 66, 1357-1363.

Saco-Pollitt, C., Pollitt, E. y Greenfield, D. (1985). The cumulative deficit hypothesis in the light of cross-cultural evidence. International Journal of Behavioral Development, 8, 75-97.

Sameroff, A. (1994). Ecological perspectives on longitudinal follow-up studies. En S.L. Friedman y H.C. Haywood (Eds.), Developmental follow-ups. Concepts, domains and method (pp. 45-64). Nueva York: Academic Press.

Susser, M. y Susser, L. (1996). Choosing a future for epidemiology: I. Eras and paradigms. American Journal of Public Health, 86, 668-673. Wachs, T. (1995). Relation of mild-to-moderate malnutrition to human development: Correlational studies. Journal of Nutrition, 2245S-2254S.

Wachs, T. (1995). Relation of mild-to-moderate malnutrition to human development: Correlational studies (ob. cit.). 
El desarrollo humano como proceso probabilístico: lecciones de treinta años de...

Winnick, M. y Noble, A. (1966). Cellular response in rats during malnutrition at various ages. Journal of Nutrition, 89, 300-306.

Winnick y Rosso (1969). Head circumference and cellular growth of the brain in normal and marismic children. Journal of Pediatrics, 74, 774778. 